\title{
Médiévales
}

Langues, Textes, Histoire

55 | automne 2008

Usages de la Bible

\section{Les commentaires latins et français aux Prophetie Merlini de Geoffroy de Monmouth (XII ${ }^{\mathrm{e}} \mathrm{XV}^{\mathrm{e}}$ siècle)}

Latin and French Commentaries on Merlin's Prophecies (twelfth-fifteenth century).

\section{Géraldine Veysseyre et Clara Wille}

\section{CpenEdition}

Journals

Édition électronique

URL : http://journals.openedition.org/medievales/5513

DOI : 10.4000/medievales.5513

ISSN : 1777-5892

Éditeur

Presses universitaires de Vincennes

\section{Édition imprimée}

Date de publication : 20 décembre 2008

Pagination : $93-114$

ISBN : 978-2-84292-221-4

ISSN : 0751-2708

Référence électronique

Géraldine Veysseyre et Clara Wille, «Les commentaires latins et français aux Prophetie Merlini de Geoffroy de Monmouth (x॥e-xve siècle) », Médiévales [En ligne], 55 | automne 2008, mis en ligne le 20 mars 2011, consulté le 01 mai 2019. URL : http://journals.openedition.org/medievales/5513 ; DOI : $10.4000 /$ medievales.5513

Ce document a été généré automatiquement le 1 mai 2019.

Tous droits réservés 


\title{
Les commentaires latins et français aux Prophetie Merlini de Geoffroy de Monmouth $\left(\mathrm{XII}^{\mathrm{e}}-\mathrm{XV}^{\mathrm{e}}\right.$ siècle)
}

\author{
Latin and French Commentaries on Merlin's Prophecies (twelfth-fifteenth \\ century).
}

Géraldine Veysseyre et Clara Wille

1 Les Prophetie Merlini, composées par Geoffroy de Monmouth vers 1135, sont l'un des rares textes profanes $d u \mathrm{XII}^{\mathrm{e}}$ siècle qui ait eu le privilège d'être commenté par les docteurs contemporains ${ }^{1}$. Elles constituent le centre de l'Historia regum Britannie, dans laquelle est retracée l'histoire du peuple breton : à partir de l'éponyme Brut qui, après la chute de Troie, arrive sur l'île, Geoffroy y égrène une succession ininterrompue de rois bretons en passant par Arthur jusqu'à Cadvalladre, dernier roi breton mort en 689. Merlin, le célèbre prophète autochtone, apparaît au moment crucial où le mauvais roi et usurpateur du trône, Vortegirn, déclenche la guerre civile parmi les Bretons. Merlin profère alors une longue prophétie sur le destin de son peuple qu'il clôt sur une vision apocalyptique.

Dès leur parution, les Prophetie Merlini connaissent un succès retentissant et elles sont immédiatement recopiées, glosées, commentées et traduites, avec ou sans l'ensemble de l' Historia regum Britannie. Nous possédons encore environ 250 manuscrits de l'Historia qui comportent presque tous les Prophetie $^{2}$; s'y ajoutent entre 80 et 100 manuscrits où les vaticinations du prophète, copiées indépendamment de l'Historia, prennent la forme d'un Libellus Merlini ${ }^{3}$. En outre, les prophéties ont été insérées intégralement dans d'autres chroniques $^{4}$ et de très nombreux textes citent des Prophetie particulières. Enfin, les prophéties ont été traduites et commentées en français ${ }^{5}$, ce qui sera l'objet de la deuxième partie de cet article.

Par commodité, les éditeurs modernes ont divisé les Prophetie Merlini en 74 unités ${ }^{6}$, au sein desquelles on distingue deux parties. La première - prophéties 1 à 12 - consiste en des prophéties ex eventu qui englobent le temps historique à partir de Merlin, au v viècle, jusqu'à la publication du Libellus Merlini par Geoffroy, en 1135 environ, et elle s'achève sur 
la mort du grand roi anglo-normand Henri $\mathrm{I}^{\mathrm{er}}$ en 1135. La deuxième partie, plus développée - prophéties 13 à 74 -, comporte des prophéties ante eventum. Jusqu'à la prophétie 20, où est annoncé le retour au pouvoir des Bretons, elle continue à prédire sous des symboles animaliers une succession de rois normands; mais à partir de la prophétie 21, les règnes des animaux et des monstres se précipitent et les Prophetie Merlini se terminent par le bouleversement des astres et la fin des temps ${ }^{7}$.

Certes, les prophéties galfrédiennes rappellent les grandes prophéties bibliques de l'Ancien Testament par leur dessein - mettre en garde le peuple pécheur en temps de guerre -; par leur formulation - le Ve initial des prophéties funestes, par exemple -; enfin par leur structure bipartite - prophéties ex eventu, puis prophéties ante eventum à l'instar des prophéties de Balaam ou de Daniel'8. Mais leur contenu est séculier, et c'est ainsi qu'une grande partie des commentateurs les ont comprises et interprétées.

Les commentaires latins aux prophéties de Merlin

5 À ce jour, nous connaissons une vingtaine de commentaires latins, dont plusieurs sont nés dès le $\mathrm{XII}^{\mathrm{e}}$ siècle$^{9}$. Pour illustrer les explications suivantes nous avons sélectionné un corpus de six commentaires rédigés entre le $\mathrm{XII}^{\mathrm{e}}$ et le $\mathrm{XIV}^{\mathrm{e}}$ siècle (voir le tableau en annexe).

Deux familles de commentaires

6 Ces prophéties ont suscité deux types fondamentaux de commentaires. Le premier, que j'appellerai le type " anglais », est historique ( ${ }^{\circ} 1$ à 5 du tableau). Le plus grand nombre des commentateurs latins n'ont interprété que les prophéties ex eventu, les rapprochant des événements passés de l'histoire anglaise. Or puisque, du XII ${ }^{e}$ au XIV siècle, on considérait que les prophéties contenaient l'histoire passée et future selon le plan divin ${ }^{10}$, les commentateurs ne se sont pas contenté d'interpréter les prophéties qui étaient $e x$ eventu pour Geoffroy : ils ont continué à décrypter les animaux des prophéties suivantes en les comprenant comme des représentations des rois anglo-normands antérieurs à leur temps ou contemporains. Ils arrêtent donc l'exégèse à leur propre époque, le plus souvent quelque part entre les «vraies» prophéties ante eventum $n^{\circ} 13$ et 22 . Tous ces commentaires aux interprétations purement historiques sont nés en Angleterre, ce qui semble naturel: les prédictions ayant été proférées par un prophète autochtone, les commentateurs les rapportent à l'histoire et aux rois anglais.

7 À titre d'exemple, nous avons retenu parmi les commentaires de ce type un texte qui subsiste en plusieurs exemplaires et illustre ainsi le problème compliqué de la tradition de ces textes : on en trouve plusieurs versions dans les manuscrits $n^{\circ} 1$ à 5 du tableau. La plus ancienne version du type « anglais » est un commentaire marginal $\left(\mathrm{n}^{\circ} 1\right)$, qui donne des explications jusqu'à la prophétie 16 et au règne d'Henri II, mais ne nomme aucun de ses fils ; il est donc possible qu'il ait été rédigé au début du règne de ce roi, peu après 1153. Ce commentaire marginal se retrouve dans plusieurs manuscrits dont nous avons choisi un échantillon de cinq représentants ( $\mathrm{n}^{\circ} 1 \mathrm{à}$ ). La mise en page en est variable. Mais ce qui est particulièrement intéressant, c'est qu'aucun de ces commentaires ne s'arrête au même endroit des Prophetie: chacun des commentateurs a repris les explications de ses prédécesseurs, puis les a prolongées par les siennes jusqu'à sa propre époque. Ainsi ce commentaire s'arrête-t-il à Henri II dans le manuscrit de Paris $\left(\mathrm{n}^{\circ} 1\right)$; dans les manuscrits de Londres et Cambridge ( $n^{\circ} 2$ et 3), il continue jusqu'à Henri III, mort en 1272 ; enfin, dans celui de Dublin ( $\left.n^{\circ} 5\right)$, il se prolonge jusqu'à Edouard II, mort en 1327. 
8 Parmi les commentaires les plus précoces figure également un deuxième type, le commentaire intitulé Explanatio in Prophetiam Merlini, qui envisage les prophéties dans le cadre plus vaste de l'histoire du salut chrétien $\left(n^{\circ} 6\right)$. C'est le seul commentaire qui interprète toutes les prophéties, y compris celles qui sont ante eventum, jusqu'au Jugement dernier ${ }^{11}$. C'est aussi le seul commentaire continental ${ }^{12}$. Contrairement aux commentaires insulaires qui, en général, ne comprennent que quelques pages, il compte 200 feuillets ; il est donc environ vingt fois plus long que le texte original. Son contenu, les derniers événements historiques qu'il mentionne, ainsi que les rares commentaires personnels de son auteur, permettent d'en situer la composition entre 1167 et 1174 ; l'auteur en est probablement un moine de Clairvaux. J'incline à penser qu'il s'agit d'Alanus Flandrensis, Autissiodorensis episcopus, originaire de Lille et converti à la vie cistercienne par saint Bernard en 1131. D'abord moine de Clairvaux et premier abbé de Larrivour, Alain est nommé évêque d'Auxerre en 1152 ; en 1167 il se retire à Larrivour, puis à Clairvaux où il meurt en 1185 dans la cellule de son maître ${ }^{13}$.

9 Dans le prologue au commentaire des Prophetie Merlini, Alain explique sa méthode herméneutique et justifie son exégèse des paroles de Merlin en s'appuyant sur les commentaires des autorités patristiques relatifs aux personnages prophétiques de l'Ancien Testament :

Les choses que Merlin dit à propos de la rédemption du monde et de la résurrection des morts, combien elles sont vraies selon les traditions prophétiques, évangéliques et apostoliques, de sorte qu'il ne s'écarte évidemment en rien de la foi orthodoxe chrétienne ; ceci est patent pour tout lecteur".

10 Par conséquent, continue Alain, Merlin est digne d'être mis au même rang que les prophètes vétérotestamentaires :

[...]. Enfin Job ne fut ni chrétien ni juif, mais certainement païen, et cependant on ne trouvera aucun prophète qui ait prophétisé plus clairement et plus fidèlement la résurrection du Christ et celle de nous tous au dernier jour. [...] Que dirais-je de Balaam, puisque ni Moïse n'a eu honte d'ajouter ses oracles à l'Écriture Sainte, ni les maîtres de l'Église de les expliquer en tant de volumes et de les répéter assidûment dans les églises de Dieu partout dans le monde ${ }^{15}$ ?

11 En outre, Alain distingue nettement le passage des prophéties ex eventu aux vraies prophéties ante eventum. En amont, pour les prophéties ex eventu, il a d'abord recours à une clé d'interprétation historique : il continue sur cette voie jusqu'aux événements de 1170 environ et débusque, comme dernières informations historiques, des événements concernant Henri II et ses fils à la prophétie 16. Mais il est le seul commentateur à mener ensuite son exégèse jusqu'à la fin des prophéties. Dans un petit prologue au dernier livre, avant de s'attaquer à l'exégèse de la fin du monde, le théologien Alain regarde ce qui précède et ce qui suit, et répète ce qu'il a déjà souligné à plusieurs reprises, à savoir que pour expliquer les «vraies " prophéties ante eventum, il faut changer de méthode et recourir à l'interprétation des différents sens selon l'exégèse scripturaire :

Pour ce qui concerne les événements qui se réaliseront après notre époque puisque l'histoire ne prophétise pas le futur, mais raconte le passé, et que c'est une chose que d'être historien, une autre que d'être prophète -, je n'ai pas suivi le fil de l'histoire, mais je me suis appliqué à rechercher le sens des mots de Merlin : selon les tropes du langage prophétique, selon les significations des mots et les figures rhétoriques, et, avant tout, selon la nature et la propriété des choses elles-mêmes, desquelles Merlin tire les analogies, et dont il emprunte les noms pour nommer ceux qu'il ne désigne pas par leurs propres noms, et, de plus, selon le langage précis 
et la manière de parler qu'il utilise dans toute son œuvre, comme je l'ai constaté

grâces à mes investigations industrieuses $[. . .]^{16}$.

$12 \mathrm{Aux} \mathrm{XIII}^{\mathrm{e}}$ et $\mathrm{XIV}^{\mathrm{e}}$ siècles, sur le Continent, ce commentaire a exercé une influence importante sur les prophéties joaquimites ${ }^{17}$ et sur Jean de Roquetaillade ${ }^{18}$; mais pour ce qui concerne les Prophetie Merlini, aucun autre commentaire de ce type ne verra le jour. La démarche d'Alain, qui recourt pour sa lecture de la prose galfrédienne à des modèles bibliques, est donc singulière, peut-être même audacieuse. C'est en tout cas ce que l'on pourrait déduire du fait qu'au $\mathrm{XVI}^{\mathrm{e}}$ siècle, l'Explanatio in Prophetiam Merlini ait été condamnée par le concile de Trente; ce qui n'empêcha pas les protestants de l'imprimer et de la publier trois fois pendant la première moitié du XVII ${ }^{\mathrm{e}}$ siècle $^{19}$.

Une présentation variable dans les manuscrits

13 La mise en page des commentaires présente deux types principaux : le premier est celui où des gloses interlinéaires expliquent des expressions particulières, alors qu'un commentaire placé dans la marge fournit des scholies portant sur le sens des phrases où ont été soulignés les lemmes commentés ${ }^{20}$. Cette mise en page est similaire à celle de la glosa ordinaria, née plus ou moins à la même époque.

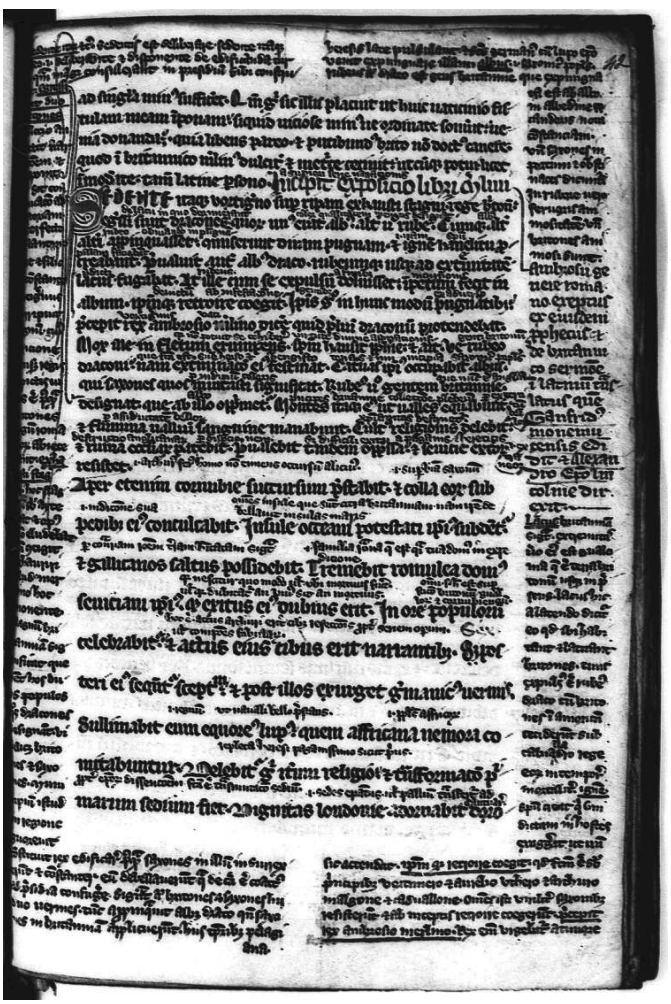

Ms Paris, BnF, lat. 6233, f. 42r : gloses interlinéaires et scholies marginales

Il existe un deuxième type de mise en page où le contenu du commentaire marginal est séparé de l'original. Il se présente comme commentaire continu où l'explication des phrases suit l'ordre des lemmes de l'original, lemmes qui sont soulignés ou singularisés par une autre convention. Ce type de commentaire est souvent ajouté dans des recueils qui comportent déjà le texte de l'Historia, comme par exemple dans les manuscrits de Lincoln et de Dublin ( $n^{\circ} 4$ et 5$)^{21}$. 


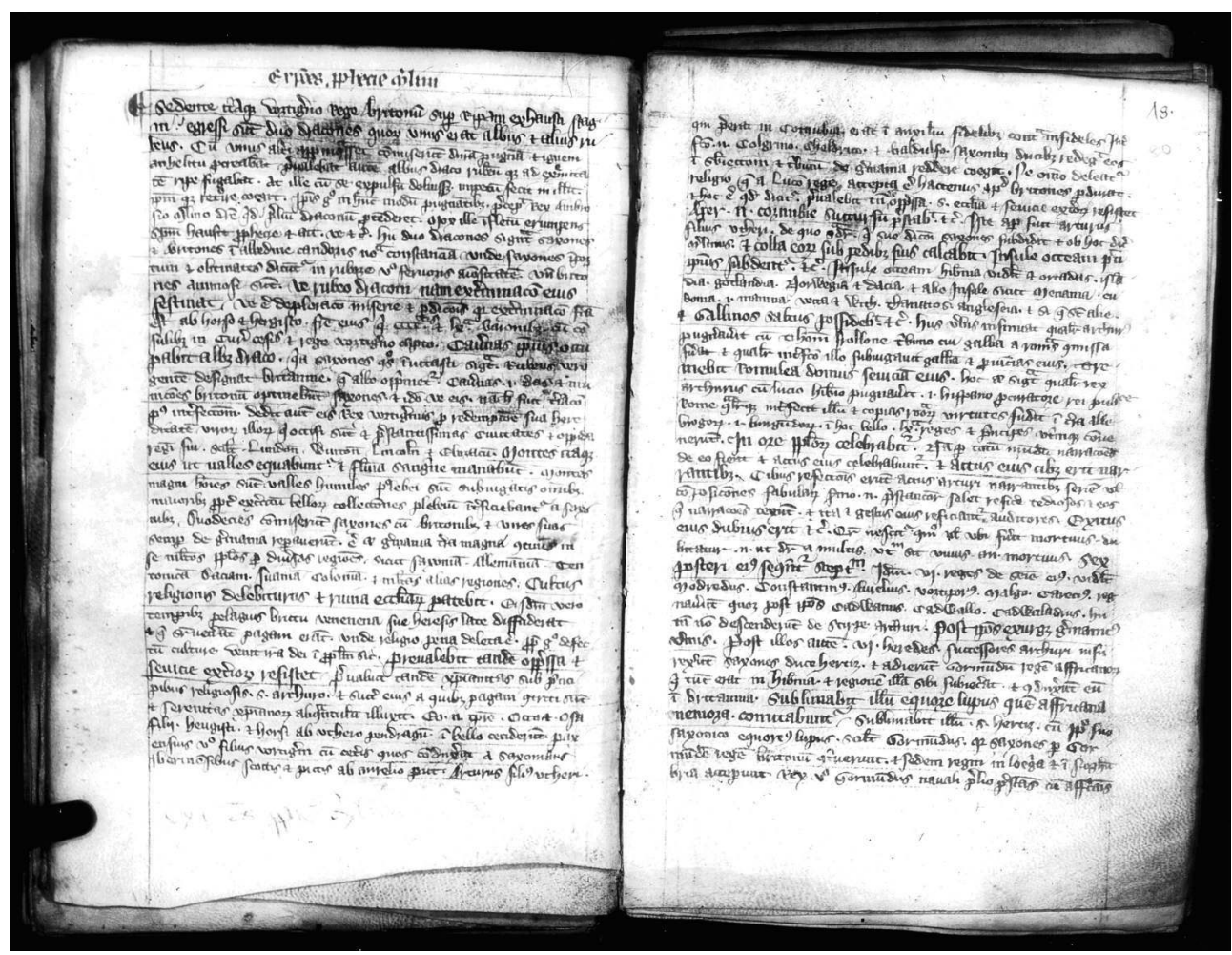

Ms Dublin, TCD 514, f. 79v

Le commentaire d'Alain constitue, là aussi, une exception par sa forme accomplie: il comporte un prologue, se voit subdivisé en sept livres et la prophétie y est fondue dans un exposé suivi. Dans le manuscrit Paris, BnF, lat. 7481, les citations des Prophetie sont marquées par les "diplés", les marques typiques des citations bibliques dans les manuscrits de commentaires des Pères de l'Église.

La prophétie $\mathrm{n}^{\circ} 12$ dans quelques commentaires latins

16 Pour effectuer une comparaison représentative, nous avons choisi un segment de la prophétie 12, la dernière des prophéties ex eventu, où l'on discerne de claires allusions à des événements historiques précis :

[1] Catuli leonis in equoreos pisces transformabuntur [2] et aquila eius super montem Arauium nidificabit ${ }^{2}$...

Cette prophétie fait allusion aux événements les plus importants du règne $\mathrm{d}^{\prime} H e n r i \mathrm{I}^{\mathrm{er}}$ : Merlin y évoque le naufrage du seul héritier d'Henri I ${ }^{\text {er }}$ et la remise en question de la succession, puis il fait allusion à sa fille, l'impératrice Mathilde, dorénavant seule héritière légitime.

Les commentaires «anglais » relatifs au deuxième segment de cette prophétie - «et aquila... » - (Paris, Londres, Cambridge et Dublin) sont très similaires :

L'aigle de celui-ci, c'est-à-dire sa fille Mathilde, à savoir l'impératrice des Romains. Le mont Aravius se trouve en pays germanique et signifie que la fille du roi fut mariée avec l'empereur des Germains et des Romains. Cette même Mathilde épousa plus tard le comte des Angevins de qui elle eut un fils ${ }^{23}$.

Ils portent tous la leçon Mons Aravius et y voient une "montagne des régions d'Allemagne ", parce que Mathilde, la fille d'Henri I ${ }^{\text {er }}$, fut mariée avec l'empereur d'Allemagne. Le commentaire de Lincoln $\left(n^{\circ} 4\right)$, est différent dans sa forme quoique 
concordant sur le fond ${ }^{24}$. Quant à Alain, le commentaire "français", il est différent à plusieurs points de vue :

Et l'aigle de celui-ci fera son nid sur le mont Morianus. Nul ne doute que l'aigle vole plus haut que les autres oiseaux. Par l'aigle du roi Henri, il < Merlin > désigne donc la fille de celui-ci, Mathilde, qui, après avoir épousé Henri, l'empereur des Romains, est elle-même devenue impératrice d'Italie, maîtresse des Romains. Par synecdoque, il désigne par le mont Morianus l'Italie, voulant faire entendre le tout par la partie. Toutefois certains livres n'ont pas mont Morianus, mais mont Avium, ce qui peut être compris de deux manières différentes selon l'équivoque de son nom. Si on l'appelle mont Avium, c'est-à-dire "le mont des oiseaux", le sens sera le suivant : que le mont des oiseaux, c'est-à-dire le mont de Jupiter, qu'on appelle par un autre nom le mont des Alpes - désignant ainsi le tout, c'est-à-dire l'Italie, par la partie -, est beaucoup plus facile à traverser et plus hospitalier pour les oiseaux que pour les hommes, et surtout pour les aigles, qui ont coutume de faire leur nid dans les rochers élevés et inaccessibles, comme le Seigneur le dit à Job. Ceux qui pensent que le mont est nommé Avius parce qu'il est avius ("sans voie"), dérivé de via ("la voie"), l'entendent ainsi : il est nommé avius, c'est-à-dire invius, non parce qu'il n'y a pas de voie du tout, mais parce que la voie elle-même est d'une certaine manière impraticable ("sans voie") [...]. Cette montagne, c'est Hannibal l'Africain qui, le premier de tous les mortels, l'a ouverte par le fer. [...] Celui-ci a donc le premier de tous les hommes, dit-on, ouvert les rochers escarpés des Alpes avec le fer et a fait une voie dans la montagne impénétrable. [...] Mais à quelle fin en sont venues la prouesse d'Hannibal, sa pompe et sa superbe? 'Il est vaincu et lui-même fuit en exil.." [...] Je sais que certains livres écrivent Mont Aravius, mont qui se trouve en tout cas en l'île d'Angleterre comme le démontrera Merlin par la suite. Par le mont Aravius est désignée l'île d'Angleterre, sur laquelle Mathilde, pour son fils, a reçu la suprématie par le don de son père $[. . .]^{*}$.

Pour le Mons Aravius de l'original, Alain présente trois leçons distinctes avec plusieurs possibilités d'interprétation à différents niveaux de sens. Il dit d'ailleurs clairement qu'il a sous les yeux un ou plusieurs commentaires où il glane ses informations - ce qui signifie qu'en 1170 différents commentaires aux prophéties avaient déjà circulé sur le Continent. D'abord, Alain dit que l'aigle, l'oiseau qui s'élève le plus haut dans son vol, désigne la fille d'Henri I ${ }^{\mathrm{er}}$, Mathilde, épouse de l'empereur, et il commente la première leçon (qu'on ne trouve d'ailleurs que chez lui), mons Morianus, qui est une synecdoque pour l'Italie. Ensuite, il s'attache à la leçon mons avium, reprenant le commentaire de Lincoln, mais donne une autre explication: mons avium signifie les Alpes. Ceci l'amène à une exégèse du sens figuré, suscitée par la figure de l'aigle et par le verset de Job: «L'aigle, à votre commandement, s'élèvera et fera son nid dans les lieux les plus élevés ${ }^{27}$ .» Il évoque donc Job, et ainsi le sens mystique, pour l'opposer à Hannibal, figure de l'Antiquité classique, afin d'introduire une longue digression à visée morale : Hannibal fut le premier à accomplir l'exploit de traverser les Alpes et à planter son étendard au centre de Rome, mais inutilement, puisque sa superbe l'a mené à une mort solitaire et atroce. Alain oppose ainsi l'humilité exemplaire de Job - élevée par Dieu - et la superbe d'Hannibal - punie par Dieu. Enfin il connaît, comme tous les autres commentaires, la leçon mons Aravius; mais, contrairement à tous les autres, il situe cette montagne en Angleterre.

21 Les Prophetie Merlini sont donc l'un des rares textes profanes du XII siècle auquel les contemporains aient consacré leurs peines herméneutiques. Ces commentaires soulignent l'impact politique et historique de ce texte. Deux familles de commentaires se dégagent : la première, née en Angleterre, concerne l'histoire anglaise. La seconde est née 
en France; nous en possédons un seul exemple, celui d'Alanus. Celui-ci est le seul à interpréter les prophéties non seulement dans le cadre de l'histoire anglaise, mais encore dans le cadre plus vaste de l'histoire du salut chrétien. Toute cette tradition des commentaires aux Prophetie Merlini a un caractère politique marqué et ne s'est continuée qu'en latin, langue des clercs et des hommes de pouvoir.

Les prophéties de Merlin en françaisUn corpus limité

Alors que les prophéties de Merlin en latin peuvent circuler indépendamment de l'Historia regum Britannie, les prophéties vernaculaires s'inscrivent toujours au sein de leur texte matrice. Quatre traductions françaises de l'Historia ont ainsi été recensées ${ }^{28}$ : le Roman de Brut de Wace (XII ${ }^{\mathrm{e}}$ siècle), en octosyllabes; l'Estoire de Brutus (XIII ${ }^{\mathrm{e}}$ siècle), traduction anonyme en prose conservée dans le seul manuscrit Paris, BnF, fr. 17177 ; les Croniques contenant les gestes des roys et princes qui regnerent en la Grant Bretaigne, traduction anonyme rédigée au début du $\mathrm{Xv}^{\mathrm{e}}$ siècle ${ }^{29}$; le Roman de Brut de Jehan Wauquelin (mi- $\mathrm{XV}^{\mathrm{e}}$ siècle), en prose. Les trois translacions en prose sont indépendantes les unes des autres; toutefois, les deux traductions anonymes entretiennent des relations plus ou moins suivies avec le travail préalable de Wace ${ }^{30}$. Ces traductions, à côté de l'abondante tradition manuscrite de l'Historia, témoignent du succès de ce texte: il a suscité l'intérêt de commanditaires de traductions jusqu'à la fin du Moyen Âge.

La présence, en son sein, des prophéties de Merlin a pu contribuer à la popularité de l' Historia; aussi la plupart des traducteurs s'astreignent-ils à transposer en français ces vaticinations alors même que la tâche est ardue. Un seul recule devant cette besogne: Wace, qui s'en tire par une pirouette :

Dunc dist Merlin les prophecies

Que vus avez, ço crei, oïes,

Des reis ki a venir esteient,

Ki la terre tenir deveient.

Ne vuil sun livre translater

Quant jo nel sai interpreter ;

Nule rien dire nen vuldreie

Que si ne fust cum jo dirreie ${ }^{31}$.

Ce faisant, Wace a frustré une part de ses lecteurs. Preuve en est que plusieurs des copistes de son Roman de Brut ont comblé cette lacune en insérant, faute de mieux, le texte latin des prophéties ${ }^{32}$. Les trois autres traducteurs, sans doute plus soucieux de satisfaire leurs commanditaires, ont accepté - exercice périlleux - de «mettre en roman» les paroles du devin.

Toutefois, la mise en valeur de ce morceau de bravoure est inégale selon les manuscrits : dans le seul exemplaire subsistant de l'Estoire des Bretons, les prophéties de Merlin sont présentées dès l'abord comme incontournables. La rubrique initiale qui annonce la teneur de l'ensemble du texte est éloquente: "Chi commence l'estoire de Brutus, de Belin, Brenes, Mellin (ses prophecies) e du roy $\operatorname{Artus}^{33}$ ». Parmi les multiples souverains qu'évoque l'Historia regum Britannie, le rubricateur n'en a sélectionné que quatre, dont les règnes constituent des temps forts du récit; Merlin est le seul personnage non royal qui lui ait semblé digne d'être mentionné. Aussi son nom est-il suivi d'une précision légitimant sa présence au sein du titre initial : c'est en tant qu'énonciateur des prophéties que Merlin y est cité. Ainsi, cet extrait saillant est mis sur le même plan que les règnes célèbres qui servent au texte de figures de proue. Le rubricateur du manuscrit BnF, fr. 2806, n'a pas mis en valeur les prophéties d'une manière aussi nette, dès l'orée de cette chronique; pour autant, dans la rubrique qui ouvre le chapitre intérieur où elles sont 
traduites, il les qualifie de « merveilleuses et haultes » (f. 39vb). Peut-être cette discrétion est-elle liée au même présupposé que le titre évoqué ci-dessus: la présence des vaticinations au sein de l'Historia étant notoire, celles-ci peuvent aussi bien être signalées sobrement que prendre une portée publicitaire. Dans tous les cas, elles apparaissent, au sein de l'ouvrage de Geoffroy, comme un maillon si essentiel que ne pas les traduire mérite d'être justifié ; et que leur présence au sein du texte a pu servir d'appât incitant à faire lire l'ensemble de l'ouvrage.

Cela peut paraitre surprenant, au moins pour le lecteur moderne, au vu de l'obscurité de ces prophéties, même une fois transposées en langue vernaculaire. De fait, sans que cette impression de lecture ait alors été dirimante - au contraire, elle apparaissait comme un gage de la crédibilité des prédictions ainsi cryptées -, cette difficulté semble avoir été ressentie dès le Moyen Âge: si une majorité des traductions de ces prophéties sont accompagnées de gloses ${ }^{34}$, c'est que leurs auteurs ou certains de leurs lecteurs ont jugé bon, tout comme leurs homologues du domaine latin, de les éclairer de commentaires exégétiques. Ainsi les gloses romanes sont-elles suscitées par une même attitude face aux prophéties de Merlin que leur pendant latin: les prophéties sont lues comme authentiques et délicates à interpréter, et comparables en cela aux écrits classiques ou à la Bible. C'est pourquoi elles stimulent volontiers les exégètes et se chargent ainsi de gloses dans l'Estoire des Bretons, voire sont intégrées au sein de commentaires organiques englobants dans les Croniques...

Deux questions toutefois se posent pour ces gloses romanes que ne suscitent ni les commentaires de la Vulgate, ni ceux qui accompagnent les prophéties latines : l'auteur de ces gloses est-il le traducteur lui-même, ou un remanieur intervenu a posteriori ? Et quel que soit l'auteur de ces gloses, ont-elles été rédigées ex ingenio ou bien tirées du ou des manuscrits latins qui ont servi de source au traducteur?

L'auteur des gloses qui accompagnent la traduction anonyme du XIII ${ }^{\mathrm{e}}$ siècle demeure presque impossible à cerner en raison de la forme de son commentaire : celui-ci, ponctuel et impersonnel dans sa formulation, pourrait fort bien avoir été ajouté dans un second temps par un intervenant autre que le traducteur. L'auteur de ce commentaire est d'autant plus difficile à situer que, loin de gloser les prophéties ex ingenio, il reprend à son compte des interprétations répandues parmi les gloses latines, particulièrement insulaires. D'ailleurs, alors que le texte comme la copie de l'Estoire des Bretons datent du XIII siècle, le commentateur ne décèle dans les prophéties de Geoffroy que l'annonce d'événements antérieurs à $1128^{35}$. Son exégèse, entièrement tributaire de gloses latines courantes, ne dévoile donc en rien le contexte dans lequel il travailla. Seule certitude, le copiste du manuscrit BnF, fr. 17177 a été sensible au caractère hétérogène des gloses présentes : il a ressenti le besoin de les distinguer du corps de la prophétie et, tout en les inscrivant au sein de la justification, il les a rubriquées, accentuant ainsi le caractère parenthétique que la syntaxe leur conférait déjà. Par leur forme, prophétie et glose sont donc dans ce cas clairement distinctes, sans qu'il soit possible de déterminer si les deux niveaux sont attribuables à deux rédacteurs successifs ou si le traducteur lui-même, ayant fondé son travail sur une Historia latine glosée, a mis en roman les commentaires en même temps que le texte.

Dans les Croniques [...] des roys et princes qui regnerent en la Grant Bretaigne, l'identité du traducteur et du glossateur est plus nette. En effet l'entrelacement syntaxique qui unit traduction et interprétation est étroit. Les deux niveaux y sont si indissociables que l'on ne peut guère imaginer une élaboration en deux temps : si le commentateur s'est fondé 
sur une traduction préalable dont nous aurions perdu tous les exemplaires non glosés, il se l'est tellement appropriée qu'on peut lui concéder au moins le statut de remanieur. Ses sources, en revanche, demeurent obscures: ne connaissant pas les gloses latines insulaires, il scrute le texte de Geoffroy pour y déceler des indices concernant l'issue de la guerre de Cent ans. Du fait qu'il les interprète en puisant avant tout dans sa propre expérience, mais aussi dans des sources livresques assez diffuses, il dévoile quelque peu le contexte dans lequel il travailla: le contenu de ses interprétations révèle qu'il est continental - il déteste les Anglais - et travaille assurément après 1356, sans doute dans le premier quart du $\mathrm{Xv}^{\mathrm{e}}$ siècle $^{36}$.

Quels que soient les auteurs et les sources de ces gloses vernaculaires, leurs méthodes méritent d'être confrontées à celles qui se déploient dans les commentaires accompagnant les prophéties de Merlin latines, mais aussi dans d'autres types de gloses, notamment bibliques.

Deux démarches herméneutiques au sein de la prophétie ${ }^{\circ} 12$

31 La vérité cryptée que sont censées receler les prophéties de Merlin relève de domaines fluctuants selon les interprètes. Certains, qui poursuivent la lecture amorcée par Geoffroy lui-même, y voient l'annonce de faits historiques; d'autres s'efforcent d'en tirer un enseignement moral.

Wace, même s'il omet les prophéties, en ébauche une analyse globale qui suffit à montrer dans quelle direction se projetteraient ses commentaires s'il s'aventurait à en proposer. Merlin y annoncerait en effet : " [1]es reis ki a venir esteient, / Ki la terre tenir deveient ${ }^{37}$ " . Faute de se faire lui-même exégète, Wace suggère là une clé de lecture ciblée. Pour lui, ces prophéties concernent la Grande-Bretagne - la formule la terre, avec son article défini à valeur de notoriété, est univoque - ; et même, de manière plus restreinte, les seuls titulaires de la royauté anglaise, donc les événements politiques de premier plan. Wace emploie-t-il l'imparfait pour laisser entendre que les événements annoncés par la prophétie se sont déjà accomplis au moment où il écrit? Il s'agirait d'un mensonge, les prophéties se prolongeant bien au-delà du segment où est évoquée la royauté du XII siècle ${ }^{38}$. Cette hypothèse n'en est pas moins vraisemblable: une telle manipulation, difficile à percer à jour en l'absence des prophéties elles-mêmes, était propre à modérer la curiosité de ses lecteurs. Si le domaine de pertinence de ces vaticinations relève déjà, au milieu du XII ${ }^{e}$ siècle, du futur dans le passé, elles ne sauraient rien apprendre à ses contemporains qu'ils ne sachent déjà. Il n'est pas impossible que la feinte modestie du traducteur ${ }^{39}$ recouvre une bonne dose de prudence politique ${ }^{40}$. Mais il y a plus: non content de censurer les propos de Merlin - il en dévoile tout juste assez pour indiquer qu'il souscrit à la lecture historique amorcée par Geoffroy -, Wace semble soucieux de détourner son public d'un extrait qu'il présente comme galvaudét1 et désormais périmé, au moins dans sa dimension divinatoire. En somme, de prophétiques, ces paroles seraient désormais devenues historiques ; il y avait là de quoi émousser leur pouvoir d'attraction.

Le commentateur anonyme du XIII ${ }^{e}$ siècle accorde aux prophéties de Merlin la même portée historique que Wace, et il privilégie lui aussi l'histoire bretonne. Mais il ne se contente pas d'une brève allusion à cette clé de lecture : il y a effectivement recours. Toutefois, il semble conscient des difficultés que recèlent les prophéties : renonçant à toute démarche exégétique singulière, il reprend en les traduisant des gloses latines insulaires $^{42}$. L'explication qu'il fournit pour la prophétie ${ }^{\circ} 12$ le montre assez : 
[1] [f. 97va] Li chaël dou lyon seront noyé em puison de mer (Guilliaumes et

Richars, li fiex le roy Henri, furent noiéz en mer, si les mengierent li poisson)

[2] et s'aigle fera nit sor le mont d'Araine (c'est sa fille) ${ }^{\text {is }}$.

latine - coïncide exactement avec celle de bien des commentaires latins ${ }^{44}$. Autre reprise directe à la tradition savante insulaire : l'élucidation de l'aigle (aquila) coïncide avec celle de la plupart des commentaires latins, qui s'accordent à y voir une figure de Mathilde, la fille d'Henri ${ }^{\text {er }}$ Beauclerc ${ }^{45}$. Toutefois, contrairement à ce qui se passe pour ses deux frères noyés, l'auteur du commentaire roman ne précise pas le prénom de la future impératrice. Que peut-on en déduire quant aux sources de ce commentaire vernaculaire? Qu'il disposait sans doute, à propos de l'aigle, d'un commentaire latin plus lapidaire que tous ceux qui ont été sélectionnés par la présente étude - sans doute un commentaire interlinéaire ${ }^{46}$. Comme ces gloses, qu'on devine resserrées, ne lui permettaient guère de nourrir sa volonté pédagogique, pourtant sensible ailleurs, le commentateur vernaculaire s'avère incapable d'expliciter le prénom de cet aigle afin de donner à son identification le même degré de précision que pour les lionceaux engloutis. N'ayant lu ni Henri de Huntingdon, ni d'autres historiens traitant de la même période, et connaissant donc mal l'histoire insulaire du $\mathrm{xII}^{\mathrm{e}}$ siècle, il est tributaire d'un précédent commentaire latin pour aborder l'histoire bretonne du siècle précédent. D'ailleurs, dès lors qu'il ne dispose plus de telles gloses latines, ses propres gloses se tarissent ${ }^{47}$. Dans tous les cas, s'en tenant à un seul niveau de lecture, il voit dans les prophéties le récit d'événements déjà accomplis de l'histoire anglaise ; sa démarche est donc parallèle à celle des exégètes qui, suivant le sens littéral, voient dans le texte biblique une histoire nationale du peuple hébreu ${ }^{48}$.

Le commentaire des prophéties tel qu'il figure dans la traduction anonyme $\mathrm{du} \mathrm{xv}^{\mathrm{e}}$ siècle a des sources plus obscures. Paradoxalement, si ce commentaire est si prolixe, c'est qu'il n'est pas fondé sur les gloses latines qui pouvaient guider notre glossateur du XIII ${ }^{\mathrm{e}}$ siècle. Aussi, contraint à innover, l'auteur des Croniques... a recours en alternance aux différentes voies d'interprétation qui s'ouvrent à tout exégète, qu'il entreprenne d'expliquer la Bible ou un texte classique ${ }^{49}$. La lecture qu'il fait du début de la prophétie $\mathrm{n}^{\circ} 12$ semble révélatrice de sa méthode :

[1] Et puis dit Merlin: "Et si seront les chas du lion transforméz en maritains poissons." Par les chas, qui sont malignes bestes plaines d'astucies prodittoires, car ilz festient leurs maistres de la queue et leur groussent des dens, sont entendus les flateurs traiteurs, qui tousjours evoquent par blandissemens prodittoires le cuer du lion a mal faire. Si seront muéz en poissons marins, car par leurs vices ilz seront exilliéz de leur païs et devendront excumeurs de mer, c'est a dire larrons de mer. Si seront prins et gettéz en la mer, et la seront devoréz des poissons qui les mengeront so.

Il ne connaît pas les commentaires insulaires, unanimes en ce point pour déceler l'allusion - prévue par Geoffroy lui-même - au naufrage des enfants royaux. En conséquence, notre glossateur échafaude ex ingenio et avec plus ou moins de rigueur des explications enchaînées dont les rapports avec la prophétie initiale sont bien distendus. La traduction de catuli par chat est ambiguë, voire inexacte; à moins que le mot chat, désignant parfois en moyen français un félin de petite taille, ne puisse par ce biais désigner le «petit d'un animal » ${ }^{51}$. Quoi qu'il en soit, c'est du sens le plus courant du terme que part notre commentateur pour avancer une explication morale de ce passage : le chat est bien représenté dans la littérature moralisante, notamment dans les exempla, et y est décrit comme un animal diabolique qui, du fait de son puissant instinct, s'avère 
impossible à dresser. Notre commentateur tire cet animal, imprévisible y compris pour son maître, vers une figure de mauvais conseiller royal, ramenant ainsi in extremis dans son explication le personnage du lion, représentant traditionnel de la royauté. Ce faisant, il tire un parti subjectif du portrait ambigu du chat qu'il commençait par citer: d'un animal ambivalent, il tire une figure monolithique. En outre il bascule insensiblement vers une herméneutique plus complexe, qui allie désormais lecture morale et lecture politique de la prophétie. C'est que, contrairement au commentateur du XIII ${ }^{\mathrm{e}}$ siècle, qui n'interprétait que les paroles de Merlin qu'il considérait comme accomplies, son émule du $\mathrm{xv}^{\mathrm{e}}$ siècle continue de voir dans ce texte des prophéties ante eventum. Plutôt que de chercher dans l'histoire passée des manifestations correspondant aux annonces de Merlin, il veut y lire des avertissements pour l'avenir. Ainsi la métamorphose des lionceaux en poissons constitue-t-elle à ses yeux une menace pour les mauvais conseillers de la personne royale : elle préfigurerait à la fois l'exil et la mort qui, d'après Merlin, les guette.

Les méthodes et les objectifs de ce glossateur sont donc originales : faisant abstraction du contexte de la prophétie vernaculaire, qui demeure matériellement inscrite au sein de l' Historia regum Britannie, il lui octroie de facto une autorité universelle, dans le temps et dans l'espace, puisqu'elle lui semble propre à mettre en garde les acteurs politiques qu'il réprouve dans la France du $\mathrm{Xv}^{\mathrm{e}}$ siècle. Cette perception du texte prophétique comme texte ouvert à une pluralité d'interprétations rappelle une caractéristique essentielle de la Bible médiévale ${ }^{52}$. Toutefois les méthodes exégétiques appliquées aux prophéties par l'auteur des Croniques... sont loin d'être aussi rigoureuses que celles de ses homologues écolâtres ou théologiens.

À lire ce commentaire, on pourrait se demander comment Wace a pu renoncer, par prudence, à traduire les vaticinations du devin: outre que leur lettre semble assez obscure pour excuser à l'avance tous les contre sens, elles sont assez touffues pour qu'il soit permis, sans trop attirer l'attention, de les commenter de manière partielle, donc partiale, et partant prudente si le besoin s'en fait sentir! Mais ce serait oublier que le contexte culturel dans lequel travaillait Wace est bien distinct de celui de son collègue du $\mathrm{XV}^{\mathrm{e}}$ siècle. Les gloses latines du XII ${ }^{\mathrm{e}}$ siècle sont en effet unanimes sur certains passages de cette prophétie et l'on décèle peut-être là une standardisation des commentaires qui n'est pas sans rappeler la fixation progressive de la glose ordinaire du texte biblique ${ }^{53}$. Or cette " glose ordinaire » des prophéties de Merlin était sans doute assez notoire dans le milieu anglo-normand du XII ${ }^{\mathrm{e}}$ siècle pour que Wace ne puisse songer à s'en affranchir.

Il faut reconnaître toutefois que ce caractère contraignant de certaines lectures historiques est le seul rapprochement précis qui s'impose entre les gloses vernaculaires aux prophéties de Merlin et l'exégèse biblique. Les traits qui les opposent prédominent largement: alors que les gloses bibliques, comme les gloses latines aux prophéties de Merlin, suscitent parfois une mise en page élaborée, nos commentaires aux prophéties vernaculaires sont toujours copiés au sein même d'une justification simple, et ne sont pas même systématiquement démarqués du reste du texte par une encre repérable. C'est que le cadre dans lequel ces commentaires ont été rédigés, tout comme le public auquel ils s'adressaient, divergent de ceux de la glossa ordinaria : il ne s'agit pas de textes savants, élaborés dans des écoles et destinés à des usages que le poids des institutions régule de fait. La présentation et le contenu des commentaires vernaculaires aux prophéties de Merlin, de même que leur présence au sein de manuscrits soignés, voire enluminés, pour le $\mathrm{BnF}$, fr. 2806, suggèrent un lectorat tout autre : des laïcs qui, séduits par la réputation 
des textes arthuriens, souhaitaient en savoir plus sur l'histoire insulaire. On ne s'étonnera pas, en conséquence, que le commentateur anonyme qui est intervenu au XIII ${ }^{\mathrm{e}}$ siècle s'en soit tenu aux gloses simples que lui fournissaient ses sources savantes; pas plus qu'on ne sera surpris de constater que son successeur $\mathrm{du} \mathrm{xv}^{\mathrm{e}}$ siècle travaille largement ex ingenio, ou du moins à partir de lectures diffuses, et qu'il compose des analyses polyvalentes. Il est vraisemblable qu'il tire parti d'une démarche intellectuelle à laquelle il a pu être initié dans un cadre scolaire, et qui lui fournit une grille de questionnement adéquate pour appréhender diverses autorités (la Bible, mais aussi certains classiques antiques); mais il s'en affranchit assez pour que ses analyses, accessibles à un lecteur moins savant, instruisent ce dernier dans divers domaines - la morale, l'histoire ou la politique. Sa démarche, en somme, est plus proche de celle du prédicateur que de l'exégète.

\section{NOTES}

1.Font aussi exception l'Architrenius de Jean de Hauvilla, l'Anticlaudianus d'Alanus ab Insulis et l'Alexandreis de Gautier de Châtillon, ainsi que quelques autres textes.

2.J.C. CRICK, The Historia regum Britannie of Geoffrey of Monmouth, III. A Summary Catalogue of the Manuscripts, Cambridge, 1989.

3.C.D. ECKHARDT, « The Prophetia Merlini of Geoffrey of Monmouth : Latin Manuscript Copies », Manuscripta, 26, 1982, p. 167-176.

4.Par exemple dans matthaei PARISIENSIs, Chronica Maiora, H.R. LUARD éd., Londres, 1872, I, p. 198-215 ; un commentaire important y est ajouté.

5.Sur la transmission des Prophetie Merlini, voir P. zUMTHOR, Merlin le Prophète, Lausanne, 1943 [Genève, 2000].

6.The Historia regum Britannie of Geoffrey of Monmouth, I. Bern, Burgerbibliothek, ms 568, N. WRIGHT éd., Cambridge, 1984, § 111-118, p. 73-84, prophéties 1-74 (abrégé en HRB par la suite). Pour les traductions des prophéties voir GEOFFROY DE MONMOUTH, Histoire des rois de Bretagne, L. MATHEY-MAILLE trad., Paris, 1993 et GOFFREDO DI MONMOUTH, La Profezia di Merlino, G. LA PLACA éd. et trad., Genève, 1990.

7.C. WILLE, « Le dossier des commentaires latins des Prophetie Merlini », dans R. TRACHSLER et al. éd., « Moult obscures paroles ». Études sur la prophétie médiévale, Paris, 2007, p. 167-184. 8.Nb 24, 3-9; Dan 10, 1-11, 39.

9.C.D. ECKHARDT, The Prophetia Merlini of Geoffrey of Monmouth, a Fifteenth Century English Commentary, Cambridge (Mass.), 1982, p. 1-15.

10.R.W. SOUTHERN, « Aspects of the European tradition of historical writing : 3 . History as prophecy », Transactions of the Royal Historical Society, 22, 1972, p. 159-180.

11.Le manuscrit Paris, BnF, lat. 6233 propose deux niveaux de commentaires. Mais seul le premier, celui des gloses interlinéaires expliquant certaines expressions, s'étend jusqu'à la fin des prophéties : le commentaire marginal qui explique le sens des phrases y est partiel.

12.Évidemment, des prophéties isolées ont très tôt été citées et commentées dans des textes continentaux (voir par exemple P. zUMTHOR, Merlin...; J.C. CRICK, « Geoffrey of 
Monmouth, prophecy and history ", Journal of Medieval History, 18, 1992, p. 357-371 ; etc.) ; mais ces emprunts sont trop partiels pour être considérés comme des commentaires au sens strict du terme.

13.Voir les arguments qui plaident en faveur de cette attribution dans C. WILLE, « Les prophéties de Merlin interprétées par un commentateur du XII ${ }^{\mathrm{e}}$ siècle », Cahiers de civilisation médiévale (sous presse). Sur Alain, voir aussi Compendium Auctorum Latinorum Medii Ævi (500-1500), I, 2, M. LAPIDGE et al. éd., Florence, 2000, p. 95.

14.Voir la note 15.

15.Paris, BnF, lat. 7481, f. 2v-4r. : « Sed et de mundi reparatione et resurrectione mortuorum, quam uera dixerit iuxta propheticas euangelicasque atque apostolicas traditiones, ita ut in nullo ab ortodoxa fide christiana deuiare uideatur cunctis legentibus patet. [...] Denique Iob nec Christianus nec Iudeus fuit, sed profecto gentilis, et tamen neminem prophetarum apertius fideliusque de Christi et nostra omnium in nouissimo die resurrectione prophetasse reperies. [...] Quid de Balaam loquar, cuius oracula nec Moysem puduit sacre legi attexere, nec magistros ecclesie tantis librorum uoluminibus explanare atque in ecclesiis Dei ubique terrarum assidue frequentare. »

16.F. 163v : « Porro de illis que post nos uentura sunt - quoniam hystorie non futura prophetant, sed que facta sunt narrant, et aliud est hystoricum esse, aliud uatem hystoriarum seriem secutus non sum, sed iuxta prophetice locutionis tropos et uerborum significationes atque figuras necnon etiam secundum rerum ipsarum naturas et proprietates, a quibus similitudines ducit, et quarum nominibus eos appellat, quos ex propria appellatione non nominat, quodque amplius est, iuxta idioma et modum loquendi, quem eum tenere per totum operis sui contextum sollerti indagatione percepi, dictorum eius intelligentiam inuestigare uacaui [...]. »

17.B. MCGINN, Visions of the End, New York, 1979, p. 180-185 ; M. REEVES, The Influence of Prophecy in the Later Middle Ages, Oxford, 1969, p. 57, 93, 521.

18.J.C. CRICK, The Historia..., III. A Summary Catalogue..., op. cit., p. 332.

19.Sous le titre de Prophetia Anglicana, Merlini Ambrosii Britanni (voir C. WILLE, « Le dossier des commentaires latins... », p. 167 et p. 168, n. 2 ; voir également M. KAUP, « Merlin, ein politischer Prophet », Cristianesimo nella Storia, 20, 1999, p. 545-578, en part. p. 551 et n. 43).

20.J. Hammer a publié le commentaire marginal du ms Paris, BnF, lat. 6233 (J. HAMMER, « A commentary on the Prophetia Merlini (Geoffrey of Monmouth's Historia regum Britannice, Book 7) », Speculum, 10, 1935, p. 3-30), ainsi que ses gloses interlinéaires (ID., « A commentary on the Prophetia Merlini (Geoffrey of Monmouth's Historia regum Britannice, Book 7, Continuation) », Speculum, 15, 1940, p. 413-431).

21.Le commentaire de Lincoln a été publié dans J. HAMMER, « Another commentary on the Prophetia Merlini (Geoffrey of Monmouth's Historia regum Britanniae, Book VII) », Bulletin of the Polish Institute of Arts and Sciences in America, 1/3, 1943, p. 589-601.

22.HRB, § 113, p. 76 : « Les petits du lion seront transformés en poissons des mers et l'aigle construira son nid sur le mont Aravius ».

23." aquila eius, id est, filia eius Matildis,. s. imperatrix Romanorum. Mons Aravius est in partibus Germaniae et significat quod desponsata est filia regis imperatori Germanorum et Romanorum. Hec eadem Matildis nupsit postea comiti Andegavensium de quo filium concepit. »

24.Lincoln, Cathedral Library, 98, f. 173r. : « aquila eius super montem Arauium nidificabit; a similitudine auium Matildis filia eius nupsit imperatori Germanie et 
Romanorum » («[...] de même que les oiseaux, Mathilde, sa fille, épousa l'empereur d'Allemagne et de Rome »).

25.D. JUNII JUVENALIS, Saturce, A.E. HovSMAN éd., Cambridge, 1938, Sat. X.159-61, p. 94.

26.Paris, BnF, lat. 7481, ff. 58v-59v : « Et aquila eius super montem Morianum nidificabit. Aquilam ceteris auibus altius uolare nemo qui dubitet. Aquilam ergo Henrici regis uocat eius filiam Mattildem, que nupta Henrico Romanorum imperatori, facta est et ipsa imperatrix Italie, domina Romanorum. Sinedochicos enim per montem Morianum designat Italiam, a parte uidelicet totum intelligi uolens. Quidam tamen codices non Morianum montem, sed montem Auium habent, quod duplici modo intelligi potest secundum ipsius nominis equiuocationem. Si enim dicas montem Auium, id est uolucrum, iste erit sensus : montem Auium, id est montem Iouis, qui alio nomine mons Alpium dicitur, quo Italia significatur, a parte scilicet totum, auibus magis quam hominibus peruium et habitabilem esse, et maxime aquilis, que in excelsis et inaccessis rupibus nidificare solent, [...] sicut Dominus loquitur ad Iob. Qui autem montem Auium ab eo, quod est auius, a uia auium dictum existimant, ita intelligunt, ut auius dicatur quasi inuius, non quod omnino uia careat, sed quod ipsa uia quodammodo inuia sit [...]. Hunc montem primus omnium mortalium Hannibal Africanus, [...] ferro aperuit [...]. Hic itaque primus hominum, ut dictum est, prerupta Alpium ferro aperuit atque in inuio uiam fecit [...]. Verum ad quem tandem finem deuenit tanta Hannibalis fortitudo, tanta pompa tantaque superbia? [...] "Vincitur idem, ipse fugit in exilium" [...]. Scio quosdam codices habere montem Arauium, qui utique infra insulam Britannie est sicut Merlinus in consequentibus demonstrabit. Per montem itaque Arauiuum insula regni Britannie significatur, cuius ipsa in filio ex dono patris optinuit principatum. »

27.Iob 39, 27 : « aut ad praeceptum tuum elevabitur aquila et in arduis ponet nidum suum.»

28.S'y ajoutent des traductions fragmentaires, incluses au sein de compilations ; par exemple la partie arthurienne des Neuf preux de Sébastien Mamerot ou l'ouverture du Roman de Perceforest.

29.Nous la citons ici d'après le manuscrit Paris, BnF, fr. 2806.

30.Sur la tradition manuscrite de ces textes et sur leurs relations avec Wace, voir G. VEYSSEYRE, "Translater" Geoffroy de Monmouth : trois traductions en prose française de l'Historia regum Britannie (XIII -XVe siècle), Thèse de doctorat, Université Paris IV-Sorbonne, 2002 (à paraitre aux éditions Droz), I, p. 11-12, et V, p. 438.

31.Wace's "Roman de Brut ». A History of the British. Text and Translation, I. ARNOLD éd. revue par J. WEISS, J. weIss trad., Exeter, 2002, p. 190, v. 7535-7542.

32.Parmi les 17 manuscrits subsistants du Roman de Brut, on en compte au moins trois qui ont été interpolés afin d'inclure en leur sein les prophéties de Merlin (voir la liste de ces exemplaires dans J. WEISS, «Introduction », dans Wace's « Roman de Brut »..., p. XXVIII ; pour plus de détails sur ces exemplaires, voir aussi G. VEYSSEYRE, « "Metre en roman" les prophéties de Merlin, voies et détours de l'interprétation dans trois traductions de l' Historia regum Britannie », dans « Moult obscures paroles »..., p. 107-166, à la p. 110, n. 14). 33.BnF, fr. 17177, f. 82vb.

34.Tel est le cas de l'Estoire des Bretons et des Croniques...

35.La dernière prophétie qu'il commente est la quinzième : il y décèle l'annonce des deux mariages successifs de Mathilde, fille d'Henri ${ }^{\text {er }}$ Beauclerc, qui épousa Henri V d'Allemagne en 1114 et Geoffroy d'Anjou en 1128. 
36.Sur les indices qui permettent de dater et de localiser ce commentaire, voir G. VEYSSEYRE, « “Metre en roman"... », op. cit., p. 109, n. 10 et p. 155.

37.Voir supra, p. 103.

38.Voir supra, p. 94.

39.Voir l'extrait cité p. 103, v. 7538-39.

40.Cette omission de Wace a fait couler beaucoup d'encre et plusieurs critiques, parmi lesquels Jean Blacker, attribuent l'abstention de Wace à sa prudence politique (J. BLACKER, « Where Wace feared to tread : on Merlin's prophecies in the reign of Henri II ", Arthuriana, 6, 1996, p. 36-52 (p. 44)). Les prophéties contenaient en effet plusieurs formules hostiles aux Normands.

41.Voir l'extrait cité p. 103, v. 7535-37.

42.G. VEYSSEYRE, «"Metre en roman"... », op. cit., p. 151-153.

43.Les portions consignées à l'encre rouge dans le manuscrit BnF, fr. 17177 sont éditées en caractères gras.

44.Voir notamment les commentaires cités supra, p. 99.

45.Voir notamment supra, p. 99.

46.Il pourrait avoir traduit des gloses analogues à celles que porte le manuscrit Exeter, Cathedral Library, 3514 ; ces gloses sont éditées dans J. HAMMER, « Bref commentaire de la Prophetia Merlini du ms 3514 de la Bibliothèque de la Cathédrale d'Exeter (Geoffrey de Monmouth, Historia regum Britanniae, 1. 7) ", dans Hommages à Joseph Bidez et à Franz Cumont , Bruxelles, 1949, p. 111-119 - nous remercions Claire Wille d'avoir attiré notre attention sur ce commentaire.

47.Il se tait définitivement à l'issue de la prophétie $n^{\circ} 21$.

48.Sur l'analyse historique de la Bible, voir notamment G. DAHAN, L'Exégèse chrétienne de la Bible en Occident médiéval (XII ${ }^{e}-\mathrm{XIV}^{e}$ siècle), Paris, 1999, p. 276-281.

49. Cette démarche polyvalente s'observe ailleurs, notamment dans l'Ovide moralisé (S. VIARRE, « Ovide au Moyen Âge », dans Dictionnaire des lettres françaises : le Moyen Âge, Paris, 1964, p. 1094-1095, et R. LEVINE, « Exploiting Ovide : Medieval Allegorizations of the Metamorphoses », Medioevo Romanzo, 14, 1989, p. 197-213).

50.BnF, fr. 2806, f. 42ra.

51.Voir la Base des lexiques du moyen français (http://www.atilf.fr/dmf/), consultée en janvier 2008.

52.G. DAHAN, L'Exégèse..., op. cit., p. 55-56.

53.Sur l'émergence de la glose ordinaire, voir notamment K. FRÖLICH et M.T. GIBSON, « Préface », dans Biblia Latina cum glossa ordinaria, Turnhout, 1992, 1, p. V-XXVIII.

\section{RÉSUMÉS}

Les prophéties de Merlin que Geoffroy de Monmouth a placées au sein de son Historia regum Britannie ont joui dès le $\mathrm{xII}^{\mathrm{e}}$ siècle d'une grande autorité. Aussi, phénomène rare pour un texte séculaire, elles ont été rapidement commentées. Il s'agit ici de voir si les méthodes qu'emploient les auteurs de ces commentaires ont partie liée avec l'exégèse biblique. La présentation 
matérielle de ces prophéties glosées oppose versions latines et versions françaises : les premières, savantes, sont surtout consignées dans des manuscrits de travail et recourent parfois à une mise en page analogue à celle de la glossa ordinaria. Les copies vernaculaires sont plus luxueuses et le commentaire, parfois rubriqué, y est toujours intégré à la justification. L'examen des voies d'interprétation déployées face à la prophétie $\mathrm{n}^{\circ} 12$ confirme d'abord ce partage des commentaires savants, apparentés à l'exégèse biblique, et des commentaires qui relèvent de la vulgarisation. Mais elle permet surtout de faire la part entre une tradition insulaire qui s'en tient à l'élucidation du sens littéral, donc à l'histoire britannique et anglaise, et une tradition continentale, qui va plus loin et se met en quête d'un sens métaphorique, souvent moral. Au-delà de ces écarts, il reste que l'exégèse biblique ne fournit pas le modèle exclusif de ces commentaires : ils relèvent d'une démarche intellectuelle qui se manifeste face à d'autres textes autorisés, qu'ils soient philosophiques, juridiques ou classiques.

Latin and French Commentaries on Merlin's Prophecies (twelfth-fifteenth century). The prophecies of Merlin, which Geoffrey of Monmouth placed at the heart of his Historia Regum Britannie, have been highly esteemed ever since he published them around 1135. In fact, soon after their publication they were already commented upon, a rare phenomenon for a secular text. The focus of this paper is to examine whether the methods employed by the authors of the commentaries followed those of biblical exegesis. In their appearance, the Latin and French manuscripts of the Prophecies of Merlin, including their commentaries, differ from each other : the former, which are learned, are found in work manuscripts and sometimes follow a layout similar to that of the glossa ordinaria. The vernacular copies, on the other hand, are more luxurious, and the commentary, sometimes marked in red, is always integrated in the text. For example, an examination of the methods of interpretation used in prophecy $\mathrm{nr} .12$ confirms that this subdivision of the learned commentary is similar to biblical exegesis and the vernacular commentaries, revealing vulgarization. However, a comparison of the Latin and vernacular texts also allows us to distinguish between the insular tradition, which restricts itself to the literal meaning, i.e. British and English history, and the continental tradition, which goes beyond in search of metaphorical and often moral signification. Apart from these differences, it remains to be said that biblical exegesis does not serve as the sole model for these commentaries: interestingly, they reveal an intellectual method which is clearly related to the one used in other authorised texts, be they philosophical, legal or classical.

\section{INDEX}

Mots-clés : histoire nationale, Merlin, prophétie

Keywords : national history, prophecy

\section{AUTEURS}

\section{GÉRALDINE VEYSSEYRE}

Université de Paris IV-Sorbonne, 1 rue Victor Cousin, F-75005 Paris

\section{CLARA WILLE}

Fonds national suisse pour la recherche scientifique, Université de Zurich, Romanisches Seminar, Zürichbergstrasse 8, 8032 Zürich 